\title{
Endovascular Baroreflex Amplification for Resistant Hypertension
}

\author{
Monique E. A. M. van Kleef ${ }^{1}$ • Mark C. Bates ${ }^{2}$. Wilko Spiering ${ }^{1}$ \\ Published online: 9 May 2018 \\ (C) The Author(s) 2018
}

\begin{abstract}
Purpose of review Most hypertension devices have been designed to interrupt or modify the sympathetic nervous system, which seems to be unbalanced in hypertension. Carotid baroreceptors play a pivotal role in maintaining adrenergic balance via a direct feedback interface and would be an exceptional target for intervention. The purpose of this review is to define the role of the baroreceptor in hypertension, to examine device-based therapies targeting the baroreflex and to explore future promises of endovascular baroreflex amplification (EBA).

Recent findings In the last two decades, two therapeutic strategies targeting the carotid baroreceptor have evolved: baroreflex activation therapy (BAT) and EBA. Both therapies enhance baroreceptor activity, either directly by electrical stimulation or indirectly by changing the geometric shape of the carotid sinus and increasing pulsatile wall strain.

Summary By showing a significant, sympathetic inhibition-mediated effect on blood pressure, BAT has laid the foundation for baroreflex-targeting therapies for resistant hypertension. EBA is a less invasive therapy with promising first-in-man study results. Ongoing randomized sham-controlled trials are needed to better understand efficacy, durability, and long-term safety and define phenotypes that may most benefit from this treatment.
\end{abstract}

Keywords Resistant hypertension - Sympathetic activity $\cdot$ Baroreceptor $\cdot$ Device-based antihypertensive therapy $\cdot$ Baroreflex activation therapy $\cdot$ Endovascular baroreflex amplification

\section{Introduction}

Approximately $10-15 \%$ of all treated hypertensive patients have resistant hypertension. Resistant hypertension is defined as blood pressure (BP) that remains above target despite adequate treatment with three or more antihypertensive medications, including a diuretic, or treatment with four or more antihypertensive medications irrespective of BP status [1•].

This article is part of the Topical Collection on Hypertension and the Kidney

Electronic supplementary material The online version of this article (https://doi.org/10.1007/s11906-018-0840-8) contains supplementary material, which is available to authorized users.

Wilko Spiering

W.Spiering@umcutrecht.nl

1 Department of Vascular Medicine, University Medical Center Utrecht, Utrecht University, P.O. Box 85500, 3508, GA

Utrecht, The Netherlands

2 CAMC Research Institute and West Virginia University, Charleston, WV, USA
Patients with resistant hypertension are at increased risk of target organ damage and cardiovascular events [2]. Although contemporary hypertension best medical therapy is often effective, some patients do not respond or cannot tolerate the prescribed antihypertensive medication regimen. This unmet need led to a growing interest in device-based therapies as an alternative or adjuvant to drugs. An exceptional target for these device-based therapies is the arterial baroreflex, which plays a central role in the development as well as propagation of the hypertensive continuum [3•].

Stimulation of the carotid baroreceptor leads to increased baroreceptor firing and reduced sympathetic outflow, resulting in a decrease in BP. Baroreflex activation therapy (BAT), in which a surgically implanted electrical stimulator activates the baroreceptor on the outer carotid sinus wall, is one of the device-based therapies that exploits this feedback loop. Although some efficacy endpoints were positive in a randomized, sham-controlled trial $[4 \cdot \bullet]$, there are considerable disadvantages: the procedure is invasive, costly, has numerous adverse effects [5], and the battery needs replacement every 3 to 5 years. Endovascular baroreflex amplification (EBA), in which an endovascular device implanted inside the carotid 
sinus modulates baroreceptor activity, is a less invasive therapy targeting the baroreflex. The first safety and efficacy results of EBA are promising $[6 \bullet \cdot]$. This review will focus on baroreceptor stimulation to lower $\mathrm{BP}$ in patients with resistant hypertension. First, we will outline the problem of resistant hypertension and provide a clinical framework for the application of device-based therapies targeting the carotid baroreceptor. Thereafter, we will discuss the role of the baroreceptor in hypertension and elaborate on the two therapies targeting the baroreflex: BAT and EBA.

\section{Resistant Hypertension and Sympathetic Nervous System}

The term resistant hypertension was initially introduced to define a group of high risk patients in which screening for secondary causes of hypertension as well as intensified treatment should be considered [1•]. Resistant hypertension is defined as BP that remains above target despite treatment with three or more antihypertensive medications of different classes, including a diuretic, or treatment with four or more antihypertensive medications of different classes irrespective of BP status [7]. Prevalence of resistant hypertension ranges from 8.4 to $17.4 \%$ in patients medically treated for hypertension [8-17] and from 8.9 to $12.8 \%$ [14-17] in all hypertensive patients. The hypertension device trials have led researchers to better define "resistant hypertension." Yet, this disambiguation process has been challenging. For example, there are patients with socalled pseudo-resistant hypertension: patients who appear to be resistant to therapy due to white coat effect or nonadherence to medication. The prevalence of white coat effect (ambulatory BP measurements being normal) among patients with resistant hypertension is estimated at $35.7 \%$ [18]. Also, the prevalence of non-adherence is surprisingly high in this population. Recent studies using therapeutic drug monitoring (in urine or plasma) to assess non-adherence to antihypertensive medication report percentages of 53 to $68 \%[19,20]$.

It is important to identify pseudo-resistance early in the clinical decision-making process. Cardiovascular risk in patients with true resistant hypertension is at least two times as high compared to those with controlled ambulatory BP [2]. Therefore, classification identifies patients at higher risk and justifies treatment intensification. Moreover, this classification is crucial to distinguish the patients that may benefit from expensive and potentially harmful device-based treatments from those in which alternative strategies, i.e., improving medication adherence, should be initiated. To date, the ideal study estimating the prevalence of true resistant hypertension has not yet been conducted. However, considering the aforementioned numbers of pseudo-resistance, prevalence of true resistant hypertension will not be much higher than $5-10 \%$, which still can be extrapolated to tens or hundreds of million patients worldwide.

Resistant hypertension is characterized by increased sympathetic drive [3•]. Studies quantifying sympathetic activity by measuring muscle sympathetic nerve activity (SNA) at the peroneal nerve have shown an increase in SNA in prehypertensive stages [21]. SNA progressively increases from mild to severe hypertension [22] and is even more pronounced in patients with resistant hypertension [23•]. Increased SNA is associated with target organ damage and an increased risk of cardiovascular events [3•]. Therefore, the sympathetic nervous system has become one of the most important targets for device-based therapies in the last decade.

\section{Baroreflex Physiology}

As the baroreceptors are important modulators of SNA, they are an exceptional target for antihypertensive therapy. Arterial baroreceptors are mechanosensitive nerve fibers located in the carotid sinus, close to the bifurcation, and in the aortic arch. These nerve fibers are located in a specialized area in the lateral wall of the carotid sinus, opposite the carotid body (another neural structure involved in BP control). The carotid sinus contains more collagen and less smooth muscle cells than the innominate, making it more compliant and contributing to stretch-induced activation of the baroreceptors [24]. Baroreceptors react on stretch rather than pressure. This has been demonstrated in an experimental model of a carotid sinus imbedded in a non-distensible plaster in which baroreceptor firing remained unaffected by increases in arterial pressure [25]. Baroreceptor nerve fibers contain ion channels that are sensitive to mechanical deformation [26॰]. Increase in wall strain causes influx of sodium and calcium ions through these channels, depolarizing the nerve terminal and generating action potentials that travel along the afferent nerve [27]. The frequency of firing depends on the magnitude of deformation and the properties of the ion channels and ion pumps located at the nerve terminal. Via the glossopharyngeal and vagus nerves, these signals travel to the nucleus tractus solitarius (NTS) in the caudal medulla. The NTS is the main regulatory center of the BP that integrates multiple information from different organ systems and the cerebral cortex. This central role of the NTS in BP regulation has been demonstrated in rat experiments. Blockage of neurotransmission in the NTS strongly attenuated the baroreflex [28] and specific lesions in the NTS eliminated baroreceptor responses completely [29]. In the NTS, two signaling pathways arise: the parasympathetic and sympathetic pathway. Signals from the parasympathetic pathway travel via the nucleus ambiguous to the heart, where parasympathetic drive is increased. The signals from the sympathetic pathway travel via the caudal ventrolateral medulla, where the signal is conversed into an inhibitory 
signal, to the rostral ventrolateral medulla (RVLM) decreasing sympathetic output [30]. The RVLM is critical for maintenance of vascular tone and is the primary output nucleus for muscle SNA.

In hypertensive patients, the baroreceptor undergoes complex changes to compensate chronic high BP status. Baroreceptors in hypertensive patients fire at higher pressures compared to normotensives in order to prevent saturation of the reflex and avoid extreme vasoconstriction and tachycardia [3•]. Although the baroreflex control of heart rate (parasympathetic pathway) is impaired [22], the baroreflex control of BP (sympathetic pathway) is unaffected in hypertension [31]. Therefore, the baroreceptors retain their ability to respond to transient changes in BP. This means that there is a shift in baroreflex activation to a higher set point, in other words, the baroreceptors are reset. Although, the specific mechanisms underlying resetting of the carotid baroreflex are yet unknown, it is likely to be multifactorial process including peripheral as well as central mechanisms $[32,33]$.

Considering the aforementioned mechanisms, modulating the baroreflex to decrease sympathetic activity seems a promising strategy to lower BP. Two device-based therapies that rely on this mechanism are BAT and EBA. BAT increases baroreceptor firing rate by directly delivering electric pulses to the baroreceptor nerve endings on the outer wall of the carotid sinus. EBA relies on mechanically changing the geometric shape of the carotid sinus during cardiac systole, which increases pulsatile strain. Pulsatility is crucial since static increases in BP reset the baroreceptor immediately, where pulsatile strain attenuates baroreceptor resetting [34]. This explains the difference in BP response to conventional carotid stenting, where low BP returns to normal within a few days, and implantation of the dynamic MobiusHD device, where decrease in BP sustains.

\section{Lessons Learned from Baroreflex Amplification Therapy}

Implantation of an electrical stimulator to activate the baroreceptor was already performed in humans in the 1950s [35]. Application of this technique in patients with severe hypertension [36, 37] or angina pectoris [38] showed a significant decrease in BP and relief of angina. Despite these promising results, the interest in electrical baroreceptor stimulation as a treatment strategy for hypertension declined due to the introduction of more effective and better tolerated antihypertensive drugs, and the technical problems associated with the first electrical stimulators. It is only since the last decade that baroreceptor stimulation regained interest for treatment of patients with resistant hypertension.

The Rheos system, an implantable electrical stimulator, has been developed by CVRx and was first investigated in 11 patients undergoing elective carotid endarterectomy [39]. Unilateral electric stimulation of the carotid sinus wall during surgery decreased systolic BP from $144 \pm 9$ to $131 \pm 9 \mathrm{mmHg}$. The first permanent implantations in humans were performed in a European multicenter feasibility study (the DEBuT-HT study) that included patients with resistant hypertension (office $\mathrm{BP} \geq 160 \mathrm{mmHg}$ systolic or $\geq 90 \mathrm{mmHg}$ diastolic despite treatment with at least three antihypertensive medications, including a diuretic) between 2004 and 2007 [40•]. Firstgeneration Rheos stimulation electrodes were placed bilaterally on the outer wall of the carotid sinus and connected to an impulse generator positioned inferior to the clavicle. BP decreased by $21 / 12 \pm 4 / 2 \mathrm{mmHg}$ at 3 months $(n=37)$ and $33 / 22$ $\pm 6 / 4 \mathrm{mmHg}$ after 2 years $(n=17)$. The subsequent randomized double-blind Rheos Pivotal trial could not prove significant acute efficacy (proportion of patients that achieve at least $10 \mathrm{mmHg}$ decrease in systolic BP at 6 months) and procedural safety (serious procedure- or system-related adverse eventfree rate within 30 days of implantation) [4••]. However, the study did show a significant difference in the proportion of patients that reached systolic BP $\leq 140 \mathrm{mmHg}$ : $42 \%$ in the active treatment group and $23 \%$ in the placebo group. Moreover, systolic BP decreased by $26 \pm 30 \mathrm{mmHg}$ in the active treatment group versus $17 \pm 29 \mathrm{mmHg}$ in the placebo group, at 6 months compared to pre-implantation. Additionally, a $40 \%$ reduction of hypertensive events in the active treatment group was observed. Yet, the substantial rate of general surgical complications and nerve injury causing transient or residual symptoms as localized numbness, dysphagia, or dysphonia led to the development of a secondgeneration device: the Barostim neo. The electrodes of the Barostim neo are substantially smaller in size, and it requires unilateral implantation only.

The Barostim neo has only been investigated in nonrandomized studies so far. The first studies showed a significant systolic office BP decrease of $26 \pm 4 \mathrm{mmHg}(n=30)$, comparable with the first-generation results [41•], and systolic 24-h ambulatory BP decrease from $148 \pm 17$ to $140 \pm$ $23 \mathrm{mmHg}(n=44)$ [42] at 6 months. Forty-three percent reached systolic office BP $\leq 140 \mathrm{mmHg}$ [41•]. More importantly, considerably less system- or procedure-related events occurred compared to the first-generation device [41•, 42]. Proof of mechanism studies with first- and secondgeneration devices have confirmed that depressor response to BAT was mediated through sympathetic inhibition [5, $43 \cdot \bullet$ and have shown that physiologic regulation of the baroreflex remained unaffected [43••]. The Barostim neo is currently being investigated in a randomized double-blind clinical trial: the Nordic BAT study [44].

The aforementioned clinical trials on BAT provide the evidence that baroreflex activation is an effective strategy to lower BP in patients with resistant hypertension. However, due to the current lack of results from randomized double- 
blind clinical trials, the side effects that occur with increased energy delivery [5], the need for a surgical procedure, the high costs, and the need for battery replacement every $3-5$ years, there has been a pursuit for alternative techniques to activate the baroreflex and lower BP.

\section{Endovascular Baroreflex Amplification}

EBA relies on a passive activation of the baroreceptor by intermittingly changing the geometric shape of the carotid sinus which increases pulsatile wall stretch (Fig. 1a). The MobiusHD is a self-expanding nitinol implant that promotes this geometric shape change. The mechanism of action is not intuitive: the outward radial forces of the longitudinal nitinol struts are not active components of the device, rather the carotid bulb components within the windows of the device. In the systolic phase of the cardiac cycle, the arc of the carotid in each window of the device projects a radius that is much larger than the radius of the baseline carotid bulb without geometric change. According to the strain equation, increased wall strain or vessel stretch would be expected with each systolic phase. In the diastolic phase of the cardiac cycle, quadrants within the device windows return to the baseline exaggerated arc. Therefore, the end result of the MobiusHD is increased carotid sinus pulsatile stretch. The implant is available in three sizes: $5.00-7.00 \mathrm{~mm}, 6.25-9.00 \mathrm{~mm}$, and 8.00 $11.75 \mathrm{~mm}$ and is placed into the internal carotid artery by a specially developed delivery catheter introduced over a guidewire via the femoral artery (Fig. 1b-d).
Fig. 1 b-d Images are reproduced by permission of Vascular Dynamics, Inc. a Crosssectional view of the carotid sinus in three different situations. In the untreated vessel, the radius increases pulsatile during the systolic phase of the cardiac cycle. The MobiusHD implant changes the geometric shape of the vessel during the systole and, therefore, increases the effective radius $(\Delta r$, in red). This results in increased vessel wall strain while preserving pulsatility. The conventional carotid stent drives the carotid sinus into a static circular shape, blunting baroreceptor function. $\mathbf{b}$ The selfexpendable nitinol MobiusHD device (c) implanted in the proximal internal carotid artery. $\mathbf{d}$ The device is delivered by a specially developed delivery catheter, introduced over a guidewire via the femoral artery. e Reproduced from Peter DA, Alemu Y,Xenos M. Fluid structure interaction with contact surface methodology for evaluation of endovascular carotid implants for drug-resistant hypertension treatment. Journal of Biomedical Engineering. 2012:134;0410016. DOI: $0.1115 / 1.4006339$. Computer simulation showing circumferential and longitudinal wall stretch variation in an average carotid sinus after device implantation, plotted for the plane shown on the left

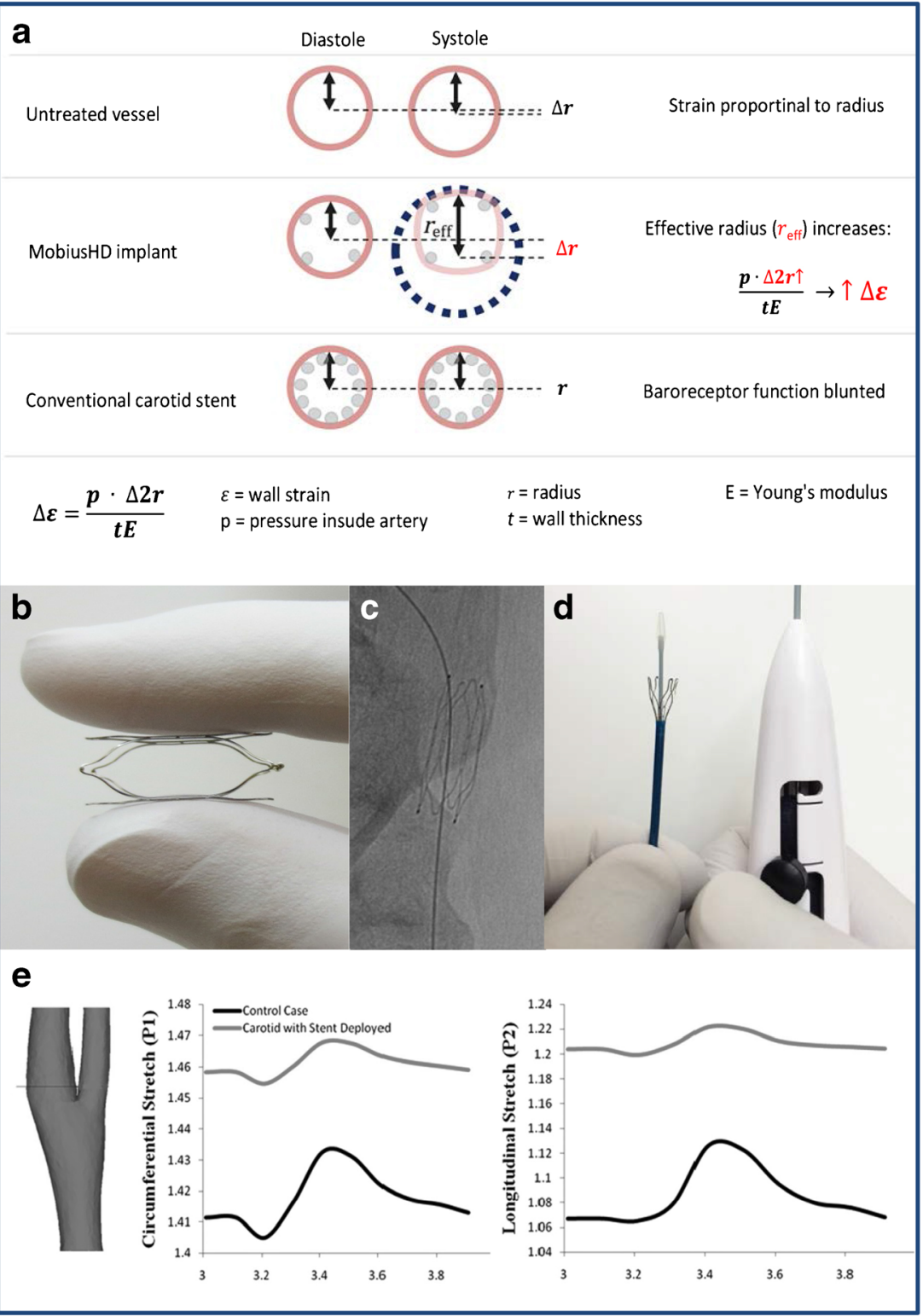




\section{Animal Studies}

The pre-clinical evaluation included several animal models to study the effect of MobiusHD implantation on hemodynamics and vessel anatomy. In a canine model, carotid baroreceptor firing rate was measured after implantation of the MobiusHD and compared to implantation of conventional self-expanding carotid stent $[6 \cdot \bullet]$. The carotid stent caused a stair step increase of carotid baroreceptor nerve activity that was decoupled with BP. In contrast, the MobiusHD not only increased immediate baroreceptor firing rate to a greater extent than the conventional carotid stent but, more importantly, showed a progressive linear increase in firing as the animal's BP was increased (Supplementary Fig. 1A). In addition, these experiments showed that MobiusHD implantation was followed by an immediate drop in BP (Supplementary Fig. 1B). In order to optimize design and ensure radial force could be programmed to reduce vessel injury or device migration through the vessel wall, several ovine carotid survival studies were performed to inform design metrics (data unpublished). Since few animals have carotid baroreceptors and an ideal surrogate for the human carotid artery is lacking, information of the effect of MobiusHD implantation on carotid biomechanics and hemodynamics largely comes from computer simulation studies.

\section{Computer Simulation Studies}

The effect of MobiusHD implantation on the carotid wall and vessel hemodynamics was studied by fluid-structure interaction combined with contact surface methodology. This technology allows for a three-way coupled dynamic interaction simulation of the endovascular device, carotid sinus, and fluid flow [45••]. Simulations were performed in two carotid models: the first representing an average carotid artery in terms of geometry and dimensions and the second representing the clinical worst-case scenario of a carotid bifurcation, devoid of a typical sinus, much smaller in dimensions, and having the internal and external carotid arteries (ICA and ECA) aligned almost parallel to each other (Supplementary Fig. 2A). The simulations showed an increase in carotid wall stretch and strain after MobiusHD deployment, affecting hemodynamics only in extreme situations. In the average model device, placement resulted in increased circumferential and longitudinal wall stretch of 2.5 and $7.5 \%$, respectively. Von Mises wall stress in the ICA increased from 198 to $305 \mathrm{kPa}$ (Supplementary Fig. 2B). The peak stress at the level of the bifurcation and the peak flow velocity in ICA and ECA did not change. In the diminutive model, circumferential and longitudinal wall stretch both increased with $6 \%$. Von Mises wall stress in the ICA increased from 60 to $90 \mathrm{kPa}$. Device deployment caused a reduction of the ECA inflow area, increasing the velocities and peak stress at the level of the bifurcation: peak stress increased from 211 to $622 \mathrm{kPa}$. Since increased stretch and stress increases baroreceptor firing rate [46•], these elevations are presumed to activate the baroreflex and reduce BP.

This simulation study additionally showed that pulsatility of the average carotid wall, although moderately attenuated, was maintained [45••] (Fig. 1e). The authors reason that the observed attenuated pulsatility is exaggerated as a result of prespecified characteristics of the device material: modeling was performed with stainless steel properties, being less elastic and stiffer than the nitinol the MobiusHD is made of. Therefore, these studies predict that the MobiusHD implantation preserves pulsatility in the carotid vessel.

\section{Results from the First-in-Man Study}

Two parallel proof-of-principle studies investigated the safety and efficacy of EBA in patients with resistant hypertension: controlling and lowering blood pressure with the MobiusHD, first in man, USA and Europe (CALM-FIM_US and CALMFIM_EUR). The first MobiusHD implantation in human was performed in 2013, Atlanta, USA. The CALM-FIM_EUR study enrolled the last patient in February 2017, and the results of this study were published in September 2017 (6••). The CALM_FIM_EUR study included 30 patients in six European centers: five in The Netherlands and one in Germany. Patients were eligible if they were 18-80 years of age; diagnosed with primary resistant hypertension; taking a stable regimen for at least 30 days with maximally tolerated doses of at least three antihypertensive drugs from different classes (one of them being a diuretic); and having a mean systolic office BP of at least $160 \mathrm{mmHg}$, mean 24-h ambulatory $\mathrm{BP}$ of at least $130 \mathrm{mmHg}$ systolic, and at least $80 \mathrm{mmHg}$ diastolic. The main exclusion criteria were hypertension secondary to an identifiable and treatable cause other than sleep apnea; any plaque or ulceration in the carotid artery or aortic arch; inadequate diameter or anatomy of the carotid vessels; history of orthostatic hypotension or syncope; atrial fibrillation; history of myocardial infarction in the past 3 months; history of cerebral vascular accident in the past 12 months; and severe renal, cardiac, or pulmonary disease. MobiusHD implantation was performed by interventionists that had at least performed 100 carotid stent implants before. Patients were treated with dual antiplatelet therapy aspirin, and clopidogrel (or equivalent), administered 3 days before up to 3 months after the procedure. Aspirin was continued indefinitely.

Patients were on average 52 years old, $15(50 \%)$ were female, $8(27 \%)$ had failed renal denervation, mean office BP was 184/109 mmHg (SD 18/14), mean 24-h ambulatory BP $166 / 100 \mathrm{mmHg}$ (SD 177/14), and mean number of antihypertensive medications 4.4 (SD 1.4). The primary endpoint was the incidence of serious adverse events (SAEs) and 
unanticipated device effects (UADEs) at 6 months. In the first 6 months, two patients had to be treated because of severe hypotension, two because of worsening of hypertension (one case in which both hypotension and hypertension occurred), and one because of dislodgement of the femoral closure device. After thromboendarterectomy, this patient developed a wound infection, which was treated by wound irrigation and antibiotics. No UADEs occurred. Most common adverse events were dizziness, musculoskeletal pain, hypotension, and groin hematoma. Not unimportantly, two patients in one center had a transient ischemic attack; fortunately, their symptoms resolved.

Mean office BP (average of two readings after $5 \mathrm{~min}$ rest, measured seated with an automated oscillometric device) decreased by $24 / 11 \mathrm{mmHg}(95 \% \mathrm{CI} 12-35 / 4-18)$ at 3 months and $24 / 12 \mathrm{mmHg}(95 \% \mathrm{CI} 13-34 / 6-18)$ at 6 months. Mean 24-h ambulatory BP decreased by $15 /$ $8 \mathrm{mmHg}(95 \% \mathrm{CI} 7-23 / 3-13)$ at 3 months and $21 /$ $12 \mathrm{mmHg}(14-29 / 7-16)$ at 6 months. This decrease was seen on top of reduction in the number of antihypertensive medication by 0.5 (IQR $1.3-0.0$ ). Unfortunately, only selfreported use of medication was assessed.

Although the sample size is small and the results of this proof-of-principal study can be highly affected by different types of bias, including regression to the mean, Hawthorneeffect, placebo effect, and observer bias, the observed decrease in BP is promising. More information on adverse effects in a larger group of patients is needed before we can reasonably state that the treatment is safe. Moreover, we need to wait for the results of the randomized, sham-controlled clinical trials to prove its efficacy.

\section{Ongoing Clinical Trials}

The CALM-START study (controlling and lowering blood pressure with the MobiusHD - studying effects in a randomized trial) is currently enrolling patients with primary resistant hypertension in planned six centers in the Netherlands and four in Germany. In this randomized sham-controlled trial, patients aged $18-70$ on a stable regimen of three to four antihypertensive medications and mean 24-h ambulatory systolic BP of 135-170 mmHg are randomized to MobiusHD implantation or sham. The primary endpoint is change in mean 24-h ambulatory systolic BP at 3 months, measured after antihypertensive medication washout. The CALM-2 study (controlling and lowering blood pressure with the MobiusHD) is a second randomized, sham-controlled multicenter trial studying the effect of EBA on BP and is planned to start recruiting patients in spring 2018. In this study, patients with resistant hypertension and mean 24-h ambulatory systolic BP of 145$200 \mathrm{mmHg}$ on a confirmed stable regimen of three to five maximally tolerated antihypertensive medications (containing at least an angiotensin-converting enzyme inhibitor or angiotensin II receptor blocker, a calcium channel blocker, and a diuretic) will be randomized. The primary endpoint is change in mean 24-h ambulatory systolic BP, obtained after observed drug intake at 6 months. These randomized trials will also provide us with more elaborate information on the efficacy and safety profile of EBA.

In addition, we are currently conducting a proof-ofmechanism study to determine the effect of EBA on SNA and baroreceptor sensitivity, in a sub-study of the CALMDIEM study (controlling and lowering blood pressure with the MobiusHD - defining efficacy markers). In this study, patients with therapy-resistant hypertension who are eligible for MobiusHD implantation undergo microneurography, cardiovascular measurements, and functional magnetic resonance imaging at baseline and 3 months, after washout of antihypertensive medications that influence sympathetic activity. Hopefully, this study will give us insight in the physiological mechanisms by which EBA reduces BP and may help us to identify which patients benefit most from treatment.

\section{Possible Benefits beyond Blood Pressure}

In addition to the effect on $\mathrm{BP}$ and the associated decrease of target organ damage, reduced sympathetic activity may have direct beneficial effects on cardiac function, renal function, and insulin sensitivity. From prospective studies in patients with primary hypertension [47] and patients with heart failure [48, 49], we know that sympathetic overactivity is associated with left ventricular hypertrophy, cardiac arrhythmias, and progressive heart failure, independent of BP. Therefore, baroreceptor stimulation may also be beneficial for patients with heart failure. A non-randomized study investigated the effect of BAT in patients with New York Heart Association functional class III heart failure and showed favorable effects on subjective (quality of life) and objective (left ventricular ejection fraction and BNP) heart failure-related outcomes [50]. A beneficial effect of sympathetic inhibition was also observed in one of our study participants, who exhibited a sharp reduction of premature ventricular complexes, 3 months after EBA.

Furthermore, sympathetic inhibition may counteract the negative effects of sympathetic outflow to the kidneys: renin release, sodium reabsorption, and renal vasculature changes [51] (including smooth muscle cell proliferation and vasoconstriction) leading to proteinuria and glomerulosclerosis [52]. Sympathetic inhibition may slow down progression of renal disease or improve renal function. In a non-randomized, single-center study, 23 patients with chronic kidney disease stage 3 or higher treated with the Barostim neo showed reduced proteinuria compared to patients who did not receive BAT [53]. However, no difference in estimated glomerular filtration rate was observed. 
Finally, EBA may play a role in regulating glucose metabolism. From earlier studies, we know that impaired glucose metabolism and hypertension often coexist. Most likely, hyperinsulinemia is preceded by increased sympathetic activity. The evidence comes from a prospective cohort study in Japan, which followed 662 normotensive and 188 borderline hypertensive age- and body mass index-matched patients for 10 years [54]. One of the explanations for the occurrence of hyperinsulinemia is that sympathetic activation leads to vasoconstriction, which lowers skeletal muscle blood flow and reduces glucose delivery to skeletal muscles [55] [56]. The effect of baroreflex stimulation on insulin sensitivity was investigated in a randomized, double-blind, cross-over trial in BP responders treated with BAT. Acute change of chronic baroreceptor stimulation did not significantly change muscular glucose delivery and insulin sensitivity [57]. Whether EBA improves cardiac function, renal function, and insulin sensitivity remains to be determined.

\section{Conclusions}

From a pathophysiological point of view, the carotid baroreceptor is an exceptional target to treat hypertension. This has been confirmed in the clinical trials evaluating BAT, showing a significant, sympathetic inhibition-mediated decrease in BP. Therefore, BAT has laid the foundation for baroreflex-targeting devices as an additive treatment for true resistant hypertension. As amplification of the baroreflex by a passive endovascular implant is less invasive, probably less costly, and does not need battery replacement every 3-5 years, EBA may be a good alternative. Although the results of the first-inman study are promising, efficacy, durability, and safety results from randomized sham-controlled clinical trials are needed before it can be implemented as a standard medical therapy. Furthermore, future research should address which patients benefit most from EBA and should examine the additional effects of sympathetic inhibition beyond lowering BP.

\section{Compliance with Ethical Standards}

Conflict of Interest M.E.A.M. van Kleef has received a research grant from Vascular Dynamics Inc.

W. Spiering is a consultant for Vascular Dynamics Inc.

M. Bates is a consultant for Vascular Dynamics Inc.

Human and Animal Rights All reported studies/experiments with human subjects performed by the authors have been previously published and complied with all applicable ethical standards (including the Helsinki declaration and its amendments, institutional/national research committee standards, and international/national/institutional guidelines).
Open Access This article is distributed under the terms of the Creative Commons Attribution 4.0 International License (http:// creativecommons.org/licenses/by/4.0/), which permits unrestricted use, distribution, and reproduction in any medium, provided you give appropriate credit to the original author(s) and the source, provide a link to the Creative Commons license, and indicate if changes were made.

\section{References}

Papers of particular interest, published recently, have been highlighted as:

- Of importance

•. Of major importance

1. Judd E, Calhoun DA. Apparent and true resistant hypertension: definition, prevalence and outcomes. J Hum Hypertens. 2014;28(8):463-8. This review defines the problem of true resistant hypertension, estimates its prevalence and underscores its associated cardiovascular risk.

2. Salles GF, Cardoso CR, Muxfeldt ES. Prognostic influence of office and ambulatory blood pressures in resistant hypertension. Arch Intern Med. 2008;168(21):2340-6.

3. Mancia G, Grassi G. The autonomic nervous system and hypertension. Circ Res. 2014;114(11):1804-14. This review lists the evidence for sympathetic overdrive in hypertension and shows that this is more pronounced with increasing severity of hypertension and end-organ damage

4.• Bisognano JD, Bakris G, Nadim MK, Sanchez L, Kroon AA, Schafer J, et al. Baroreflex activation therapy lowers blood pressure in patients with resistant hypertension results from the doubleblind, randomized, placebo-controlled rheos pivotal trial. J Am Coll Cardiol. 2011;58(7):765-73. This is the first randomized double-blind clinical trial investigating the safety and efficacy of BAT. Although negative on 2 of the 5 co-primary endpoints, it did show a significant difference in the proportion of patients in that reached blood pressure control

5. Heusser K, Tank J, Brinkmann J, Menne J, Kaufeld J, Linnenweber-Held S, et al. Acute response to unilateral unipolar electrical carotid sinus stimulation in patients with resistant arterial hypertension. Hypertension. 2016;67(3):585-91.

6.• Spiering W, Williams B, Van der Heyden J, van Kleef M, Lo R, Versmissen J, et al. Endovascular baroreflex amplification for resistant hypertension: a safety and proof-of-principle clinical study. Lancet. 2017;390(10113):2655-61. This proof-of-principle, first-in-human study showed that endovascular baroreceptor amplification with the MobiusHD device substantially lowers blood pressure with an acceptable safety profile

7. Calhoun DA, Jones D, Textor S, Goff DC, Murphy TP, Toto RD, et al. Resistant hypertension: diagnosis, evaluation, and treatment. A scientific statement from the American Heart Association professional education committee of the council for high blood pressure research. Hypertension. 2008;51(6):1403-19.

8. Giannattasio C, Cairo M, Cesana F, Alloni M, Sormani P, Colombo G, et al. Blood pressure control in Italian essential hypertensives treated by general practitioners. Am J Hypertens. 2012;25(11):1182-7.

9. Falaschetti E, Chaudhury M, Mindell J, Poulter N. Continued improvement in hypertension management in England: results from the health survey for England 2006. Hypertension. 2009;53(3):480-6.

10. ALLHAT Collaborative Research Group. Major cardiovascular events in hypertensive patients randomized to doxazosin vs chlorthalidone: the antihypertensive and lipid-lowering treatment to prevent heart attack trial (ALLHAT). JAMA. 2000;283(15):1967-75. 
11. Pepine CJ, Handberg EM, Cooper-DeHoff RM, Marks RG, Kowey $\mathrm{P}$, Messerli FH, et al. A calcium antagonist vs a non-calcium antagonist hypertension treatment strategy for patients with coronary artery disease. The international verapamil-trandolapril study (INVEST): a randomized controlled trial. JAMA 2003;290(21): 2805-2816.

12. Jamerson K, Weber MA, Bakris GL, Dahlof B, Pitt B, Shi V, et al. Benazepril plus amlodipine or hydrochlorothiazide for hypertension in high-risk patients. N Engl J Med. 2008;359(23):2417-28.

13. Dahlof B, Devereux RB, Kjeldsen SE, Julius S, Beevers G, de Faire $\mathrm{U}$, et al. Cardiovascular morbidity and mortality in the losartan intervention for endpoint reduction in hypertension study (LIFE): a randomised trial against atenolol. Lancet. 2002;359(9311):995-1003.

14. McAdam-Marx C, Ye X, Sung JC, Brixner DI, Kahler KH. Results of a retrospective, observational pilot study using electronic medical records to assess the prevalence and characteristics of patients with resistant hypertension in an ambulatory care setting. Clin Ther. 2009;31(5):1116-23.

15. Persell SD. Prevalence of resistant hypertension in the United States, 2003-2008. Hypertension. 2011;57(6):1076-80.

16. Egan BM, Zhao Y, Axon RN, Brzezinski WA, Ferdinand KC. Uncontrolled and apparent treatment resistant hypertension in the United States, 1988 to 2008. Circulation. 2011;124(9):1046-58.

17. Sim JJ, Bhandari SK, Shi J, Liu IL, Calhoun DA, McGlynn EA, et al. Characteristics of resistant hypertension in a large, ethnically diverse hypertension population of an integrated health system. Mayo Clin Proc. 2013;88(10):1099-107.

18. de la Sierra A, Segura J, Banegas JR, Gorostidi M, de la Cruz JJ, Armario P, et al. Clinical features of 8295 patients with resistant hypertension classified on the basis of ambulatory blood pressure monitoring. Hypertension. 2011;57(5):898-902.

19. Jung O, Gechter JL, Wunder C, Paulke A, Bartel C, Geiger H, et al. Resistant hypertension? Assessment of adherence by toxicological urine analysis. J Hypertens. 2013;31(4):766-74.

20. de Jager RL, van Maarseveen EM, Bots ML, Blankestijn PJ, investigators S. Medication adherence in patients with apparent resistant hypertension: findings from the SYMPATHY trial. Br J Clin Pharmacol. 2018;84(1):18-24.

21. Smith PA, Graham LN, Mackintosh AF, Stoker JB, Mary DA. Relationship between central sympathetic activity and stages of human hypertension. Am J Hypertens. 2004;17(3):217-22.

22. Grassi G, Cattaneo BM, Seravalle G, Lanfranchi A, Mancia G. Baroreflex control of sympathetic nerve activity in essential and secondary hypertension. Hypertension. 1998;31(1):68-72.

23. Grassi G, Seravalle G, Brambilla G, Pini C, Alimento M, Facchetti $\mathrm{R}$, et al. Marked sympathetic activation and baroreflex dysfunction in true resistant hypertension. Int J Cardiol. 2014;177(3):1020-5. Study that showed marked sympathetic activation and impaired baroreflex control in patients with resistant hypertension

24. Rees PM, Jepson P. Measurement of arterial geometry and wall composition in the carotid sinus baroreceptor area. Circ Res. 1970;26(4):461-7.

25. Hauss WH, Arndt T, Derlam G, Kemper F. Effect of carotid sinus stimulation on the electroencephalogram. Z Kreislaufforsch. 1954;43(23-24):831-40.

26. Hajduczok G, Chapleau MW, Ferlic RJ, Mao HZ, Abboud FM. Gadolinium inhibits Mechanoelectrical transduction in rabbit carotid baroreceptors - implication of stretch-activated channels. J Clin Investig. 1994;94(6):2392-6. Study in rabbits showing that blockage of stretch-sensitive channels reduced baroreceptor activity

27. Chapleau MW, Li Z, Meyrelles SS, Ma XY, Abboud FM. Mechanisms determining sensitivity of baroreceptor afferents in health and disease. Ann N Y Acad Sci. 2001;940:1-19.

28. Ohta H, Talman WT. Both Nmda and non-Nmda receptors in the Nts participate in the baroreceptor reflex in rats. Am J Physiol-Reg I. 1994;267(4):R1065-R70.
29. Reis DJ. The brain and hypertension - reflections on 35 years of inquiry into the neurobiology of the circulation. Circulation. 1984;70(5):31-45.

30. Ross CA, Ruggiero DA, Joh TH, Park DH, Reis DJ. Adrenaline synthesizing neurons in the rostral ventrolateral medulla - a possible role in tonic vasomotor control. Brain Res. 1983;273(2):356-61.

31. Mancia G, Ludbrook J, Ferrari A, Gregorini L, Zanchetti A. Baroreceptor reflexes in human hypertension. Circ Res. 1978;43(2):170-7.

32. Chapleau MW, Hajduczok G, Abboud FM. Peripheral and central mechanisms of baroreflex resetting. Clin Exp Pharmacol Physiol Suppl. 1989;15:31-43.

33. Victor RG. Carotid baroreflex activation therapy for resistant hypertension. Nat Rev Cardiol. 2015;12(8):451-63.

34. Mendelowitz D, Scher AM. Pulsatile sinus pressure changes evoke sustained baroreflex responses in awake dogs. Am J Phys. 1988;255(3 Pt 2):H673-8.

35. Carlsten A, Folkow B, Grimby G, Hamberger CA, Thulesius O. Cardiovascular effects of direct stimulation of the carotid sinus nerve in man. Acta Physiol Scand. 1958;44(2):138-45.

36. Bilgutay AM, Lillehei CW. Surgical treatment of hypertension with reference to baropacing. Am J Cardiol. 1966;17(5):663-7.

37. Schwartz SI, Griffith LS, Neistadt A, Hagfors N. Chronic carotid sinus nerve stimulation in the treatment of essential hypertension. Am J Surg. 1967;114(1):5-15.

38. Braunwald E, Epstein SE, Glick G, Wechsler AS, Braunwald NS. Relief of angina pectoris by electrical stimulation of the carotidsinus nerves. N Engl J Med. 1967;277(24):1278-83.

39. Schmidli J, Savolainen H, Irwin E, Peters T, Cain C, Martin R, et al. A completely new treatment for hypertension? J Hypertens. 2004;22:S252-S3.

40. Tordoir JHM, Scheffers I, Schmidli J, Savolainen H, Liebeskind U, Hansky B, et al. An Implantable carotid sinus baroreflex activating system: surgical technique and short-term outcome from a multicenter feasibility trial for the treatment of resistant hypertension. Eur J Vasc Endovasc. 2007;33(4):414-21. This is the first clinical study investigating the Rheos device in human patients with resistant hypertension

41. Hoppe UC, Brandt MC, Wachter R, Beige J, Rump LC, Kroon AA, et al. Minimally invasive system for baroreflex activation therapy chronically lowers blood pressure with pacemaker-like safety profile: results from the Barostim neo trial. J Am Soc Hypertens. 2012;6(4):270-6. First clinical study with the Barostim neo device showing comparable blood pressure decrease and less nerve injury compared to the Rheos device

42. Wallbach M, Lehnig LY, Schroer C, Lueders S, Bohning E, Muller GA, et al. Effects of Baroreflex activation therapy on ambulatory blood pressure in patients with resistant hypertension. Hypertension. 2016;67(4):701-9.

43.• Heusser K, Tank J, Engeli S, Diedrich A, Menne J, Eckert S, et al. Carotid baroreceptor stimulation, sympathetic activity, baroreflex function, and blood pressure in hypertensive patients. Hypertension. 2010;55(3):619-26. Proof-of-mechanism study that confirmed sympathetic inhibition-mediated blood pressure decrease by BAT with the Rheos device

44. Gordin D, Fadl Elmula FEM, Andersson B, Gottsater A, Elf J, Kahan T, et al. The effects of baroreflex activation therapy on blood pressure and sympathetic function in patients with refractory hypertension: the rationale and design of the Nordic BAT study. Blood Press. 2017;26(5):294-302.

45.• Peter DA, Alemu Y, Xenos M, Weisberg O, Avneri I, Eshkol M, et al. Fluid structure interaction with contact surface methodology for evaluation of endovascular carotid implants for drug-resistant hypertension treatment. J Biomech Eng-T Asme. 2012;134(4). Computer simulation study showing increased carotid wall 
stretch and strain after MobiusHD deployment, affecting haemodynamics only in extreme situations.

46. Andresen MC, Krauhs JM, Brown AM. Relationship of aortic-wall and baroreceptor properties during development in normotensive and spontaneously hypertensive rats. Circulation Research. 1978;43(5):728-38. Study in normotensive and spontaneously hypertensive rats showing that increased wall stress is associated with increased baroreceptor discharge

47. Schlaich MP, Kaye DM, Lambert E, Sommerville M, Socratous F, Esler MD. Relation between cardiac sympathetic activity and hypertensive left ventricular hypertrophy. Circulation. 2003;108(5):560-5.

48. Kaye DM, Lefkovits J, Jennings GL, Bergin P, Broughton A, Esler MD. Adverse consequences of high sympathetic nervous activity in the failing human heart. J Am Coll Cardiol. 1995;26(5):1257-63.

49. Brunner-La Rocca HP, Esler MD, Jennings GL, Kaye DM. Effect of cardiac sympathetic nervous activity on mode of death in congestive heart failure. Eur Heart J. 2001;22(13):1136-43.

50. Gronda E, Seravalle G, Brambilla G, Costantino G, Casini A, Alsheraei A, et al. Chronic baroreflex activation effects on sympathetic nerve traffic, baroreflex function, and cardiac haemodynamics in heart failure: a proof-of-concept study. Eur J Heart Fail. 2014;16(9):977-83.
51. Barrett CJ. Renal sympathetic nerves - what have they got to do with cardiovascular disease? Exp Physiol. 2015;100(4):359-65.

52. Joles JA, Koomans HA. Causes and consequences of increased sympathetic activity in renal disease. Hypertension. 2004;43(4): 699-706.

53. Wallbach M, Lehnig LY, Schroer C, Hasenfuss G, Muller GA, Wachter R, et al. Impact of baroreflex activation therapy on renal function - a pilot study. Am J Nephrol. 2014;40(4):371-80.

54. Masuo K, Mikami H, Ogihara T, Tuck ML. Sympathetic nerve hyperactivity precedes hyperinsulinemia and blood pressure elevation in a young, nonobese Japanese population. Am J Hypertens. 1997;10(1):77-83.

55. Esler M, Straznicky N, Eikelis N, Masuo K, Lambert G, Lambert E. Mechanisms of sympathetic activation in obesity-related hypertension. Hypertension. 2006;48(5):787-96.

56. Lembo G, Capaldo B, Rendina V, Iaccarino G, Napoli R, Guida R, et al. Acute noradrenergic activation induces insulin-resistance in human skeletal-muscle. Am J Physiol. 1994;266(2):E242-E7.

57. May M, Ahrens J, Menne J, Haller H, Beige J, Eckert S, et al. Limited acute influences of electrical baroreceptor activation on insulin sensitivity and glucose delivery: a randomized, doubleblind, crossover clinical study. Diabetes. 2014;63(8):2833-7. 